\title{
МОДЕЛИРОВАНИЕ ОПТИМАЛЬНОГО РАЗМЕРА ЗАПАСА МАТЕРИАЛЬНЫХ ЦЕННОСТЕЙ СКЛАДА ВАГОННОГО ДЕПО
}

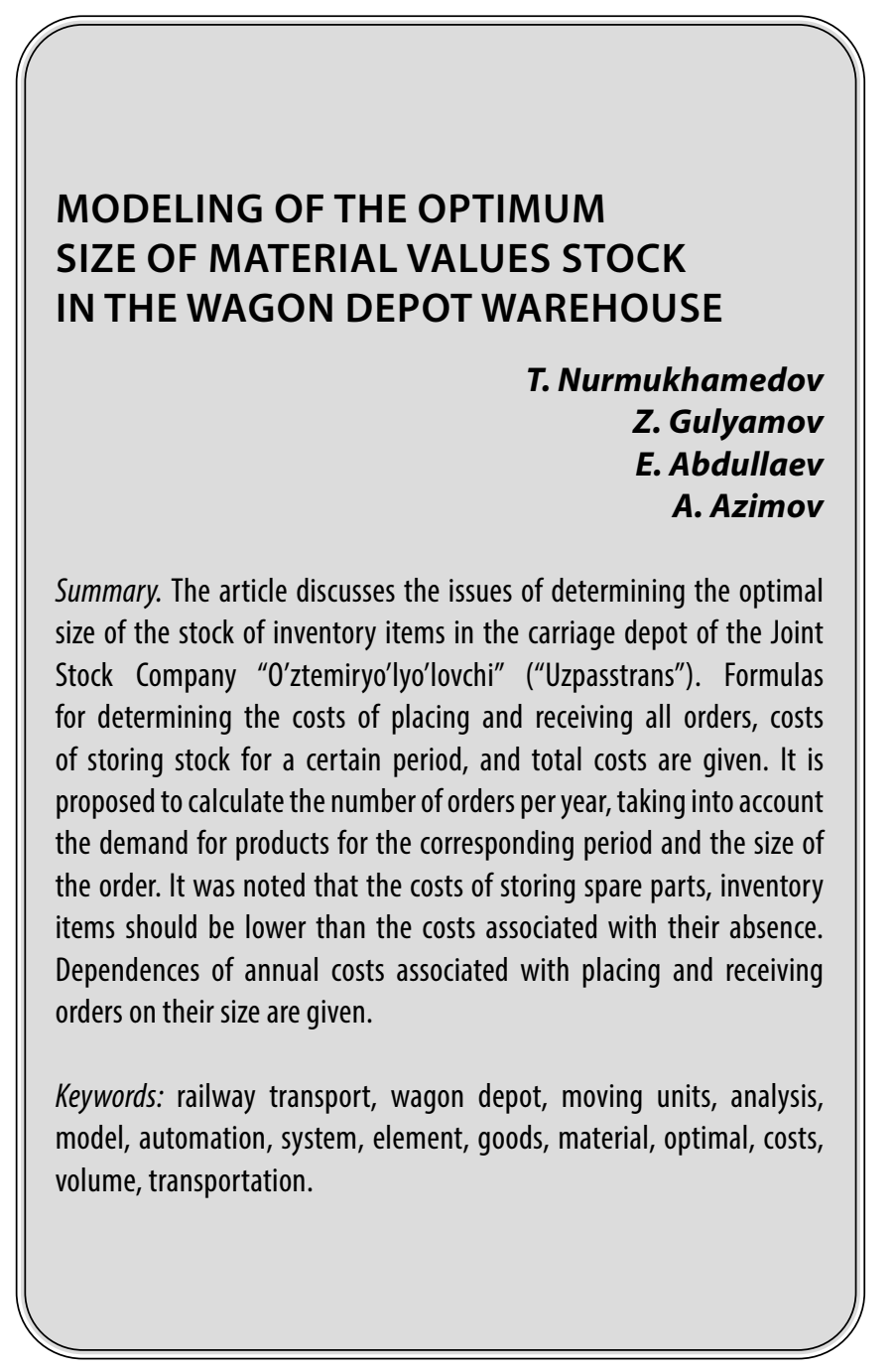

\section{Введение}

$\mathbf{0}$ сновное назначение склада вагонного депо концентрация товарно-материальных ценностей (ТМЦ), запасов, их безопасное хранение, накапливание, выдача запасных частей, комплектующих и другой продукции сотрудникам задействованных в ремонтно-экипировочных работах. Склады вагонного депо - это сложные системы и они должны строго соответствовать тем функциям, которые на них возлагаются. Организация запасов на складах вагонного депо железнодорожного транспорта (ЖТ) в конечном итоге направлена на равномерное выполнение технических и экипировочных работ, что в конечном итоге создает устойчивую работу подвижных единиц.
Нурмухамедов Толаниддин Рамзиддинович Д.т.н., профессор, Ташкентский государственный транспортный университет ntolaniddin@mail.ru

Гулямов Жавлон Нуруллаевич Старший преподаватель, Ташкентский государственный транспортный университет javlonbek1207@gmail.com Абдуллаев Элдор Саъдулла угли Ассистент, Ташкентский государственный транспортный университет eldorabdullayev@list.ru

Азимов Абдулхай Абдулатиф угли Ассистент, Ташкентский государственный транспортный университет azimovabdulhay1707@gmail.com

Аннотация. В статье рассматриваются вопросы определения оптимального размера запаса товарно-материальных ценностей в вагонном депо Акционерного общества «Узтемирйулйуловчи» («Узжелдорпасс»). Приведены формулы определения затрат на размещение и получение всех заказов, затрат на хранение запаса в течение определенного периода, общие затраты. Расчет количества заказов за год предлагается выполнять с учетом спроса на продукцию за соответствующий период и размером заказа. Отмечено, расходы на хранение запасных частей, товарно-материальных ценностей должны быть ниже расходов, связанных с их отсутствием. Приведены зависимости годовых затрат, связанных с размещением и получением заказов, от их размера.

Ключевые слова: железнодорожный транспорт, вагонное депо, подвижные единицы, анализ, модель, автоматизация, система, элемент, товары, материальный, оптимальный, затраты, объем, перевозки.

Склад вагонного депо должен [1]:

- обеспечить своевременное обслуживание пользователей по заявке и выдачей требуемых ТМЦ;

- сократить использование человеческого труда, освободить от рутинных операций;

- создать на складских помещениях необходимые условия для качественного хранения ТМЦ.

Исходя из отмеченных требований, современные складские автоматизированные информационные системы позволяют значительно снизить потери при хранении, транспортировке, сортировке и переработке товаров, минимизировать влияние человеческого фактора, рисков возникновения аварийных ситуаций работы автоматизированных технологических ком- 
плексов по хранению, транспортировке и переработке ТМЦ, снизить простои авто - и ж/д составов.

Основным показателем эффективности автоматизации складской системы, является рентабельность. Экономический результат достигается только тогда, когда планирование и реализация складской системы анализируются, учитывая интересы всего предприятия.

Место склада в логистической системе и его функции напрямую влияют на техническую оснащенность склада. Склады встречаются в различных функциональных областях логистики (снабженческой, производственной и распределительной) [2]. Железнодорожный склад можно отнести к производственному типу: связаны с обработкой груза относительно постоянной номенклатуры, поступающего и уходящего со склада с определенной периодичностью и малым сроком хранения.

Автоматизация учетных операций вагонного склада подразумевает под собой целый комплекс мер, целью которых является оптимизация, автоматизация и улучшение общего уровня эффективности его работы. Основными направлениями внедрения системы автоматизации на складе являются: оптимизация оборота ТМЦ на складе, автоматизация ведения документооборота, оптимизация работы сотрудников склада. Работа по этим направлениям позволяет оперативно контролировать остатки продукции на складе, оптимизировать расчет учетной стоимости склада, проводить более продуктивную инвентаризацию склада. Автоматизация складской логистики позволяет оптимизировать работу не только внутри самого склада, но и на предприятии в целом [3].

Логистические ИТ-системы и процесс автоматизации склада можно разделить на несколько уровней [2, 3]:

- внедрение учетных систем (как правило, ритейлеры применяют ERP или WMS);

- оптимизация складского хозяйства, за счет применения математических методов для построения модели склада и оценки эффективности складских процессов;

- системы мониторинга и трекинга;

- роботизация склада.

Первая категория систем - простые учетные системы, отражающие бизнес-события или факты и работающие в полу ручном режиме: человек заносит в систему информацию, которая распространяется по другим информационным системам, согласно заложенным правилам и настроенным бизнес-процессам. Наибольшее развитие и внедрение получила WMS-система
(Warehouse Management System - система управления складом), которая призвана автоматизировать работу складского персонала, а также обеспечить оптимизацию и контроль выполнения заданных технологических и бизнес-процессов, принятых в складском комплексе. Среди этих систем достаточно широкое развитие получили такие WMS системы как: Logistics Vision Suite, SSA Warehouse Management 4000 (EXceed ${ }^{\mathrm{TM}}$ WMS4000), Manhattan, HighJumpWarehouseAdvantage, Advantics. WM, Solvo WMS, Radio Beacon WMS, Navision, Warehouse Expert, Система\#1 WMS, ФОЛИО Логистик Склад, БУХта: Складской Комплекс и др. [4].

Разработка автоматизированных складов на основе математических методов моделирования позволяет создать эффективную систему управления складом, предиктивно смотреть на процессы, не только внутренние, но и внешние - складской двор, грузовые потоки между складами, рационально осуществлять перевозки ТМЦ из центрального хаба на локальные и т.д. Надо отметить, что математическое моделирование складской деятельности данной категории ориентировано на использование программного обеспечения (ПО) конкретного заказчика, или с использованием специализированного ПО, требующего высоких технических навыков. Склады вагонного депо по объему, запасам ТМЦ относятся к малым предприятиям, которые в настоящее время в преобладающем большинстве случаев не полностью автоматизированы. Исходя из этого вопросы рациональной организации складского учета ТМЦ вагонного депо, его информационное сопровождение для оценки и планирования, складских логистических систем является актуальной.

Вагонное депо «ВЧД-2» является одним из основных структурных подразделений Акционерного общества «Узтемирйулйуловчи», которое призвано своевременно и качественно выполнять ремонтно-экипировочные работы с подвижными единицами. При этом на складах вагонного депо стараются эффективно управлять запасами товарно-материальных ценностей (ТМЦ), комплектующих изделий, запасных частей, таким образом, что в конечном итоге положительно влияет на выравнивание, равномерную организацию перевозочного процесса. Службы материально-технического обеспечения нацелены на рост объемов запасных частей и на количественное и качественное удовлетворение потребностей работников, участвующих в перевозочном процессе, и естественно они стараются добиться увеличения запасов на всех стадиях выполнения ремонтно-экипировочных работ. Такая позиция характерна для всех производственных служб, так как высокие нормы запасов обеспечивают большую степень надежности в работе, предотвращая тем самым срывы и простои [2]. В то же время финансовое управление дороги 
стремится к сокращению затрат на организацию запасов ТМЦ до минимально возможного уровня, уменьшая тем самым затраты на их хранение.

Хотя на ЖТ содержание запасов ТМЦ сопряжено с определенными затратами, но несмотря на это пассажирская служба дороги вынуждена их создавать, так как отсутствие запасов может привести к еще большей потере прибыли в связи с простоем подвижных единиц.

Запасные части, ТМЦ, имеющиеся в запасе, должны иметь расходы на хранение, ниже расходов, связанных с их отсутствием. Только в этом случае можно создавать запасы, т.к. они будут рентабельны при их хранении [4].

Отметим основные проблемы вагонного депо, которые наблюдаются при управлении запасами:

- величина и возможная неравномерность расхода, отдаленность поставщиков, ограничения по ресурсам, способам транспортировки;

- различные виды запасов: текущие, страховые, сезонные и др.;

- большое число параметров, по которым необходимо принимать решения при управлении запасами: величина заказа, момент заказа, момент поставки, интервал времени между заказами, величина страхового запаса и др.;

- увеличение времени выполнения заказов, размещаемых в отдаленных зонах с дешевой рабочей силой.

\section{Основная часть: определение оптима^ьного размера текушего запаса}

Текущий запас, размещенный на складе вагонного депо, может иметь максимальный, средний или минимальный размер. Если пополнение запасов на складе будет осуществляться ежеквартально, то в этом случае, в зависимости от расхода и поставки размер текущего запаса меняется от максимального его значения до минимального (0 единиц). Оптимальный размер текущего запаса соответствует оптимальному значению его средней величины $\left(3_{\text {тек.ср }}\right)$, которое равно половине заказанной и доставленной партии ТМЦ. В этом случае оптимальный размер заказываемой партии ТМЦ соответствует оптимальному размеру запаса. Следовательно, необходимо решить задачу определения оптимального размера запаса. Критерием оптимума при нахождении оптимального размера запаса является минимум общих затрат за период, связанных с приобретением и хранением запаса [2-4].

Определение размера запаса товарно-материальных ценностей склада вагонного депо заключается в разработке основной модели расчета оптимального размера заказа EOQ (Economic Order Quantity), в котором в качестве критерия оптимизации принимается минимум общих затрат $C_{\Sigma}$, включающих затраты на выполнение заказов $C_{3}$ и затраты на хранение запаса на складе $C_{x}$ в течение определенного периода времени (год, квартал и т.п.):

$$
C_{\Sigma}=C_{3}+C_{x} \rightarrow \min
$$

Затраты на выполнение заказов $C_{3}$ с увеличением размера заказа уменьшаются, как правило, по гиперболической зависимости, а затраты на хранение $C_{x}$ партии поставки возрастают прямо пропорционально размеру заказа. Изменение общих затрат $\left(C_{\Sigma}\right)$, имеет вогнутый характер, что говорит о наличии минимума, соответствующего оптимальной партии заказа $\mathrm{S}_{o}$.

Для вывода формулы EOQ рассмотрим зависимость затрат $C_{3}$ и $C_{x}$ от партии заказа $S$. Определим затраты, связанные с выполнением заказа, которые определяются по формуле

$$
C_{3}=\frac{A * C_{0}}{S}
$$

где $A$ - потребность в заказываемом продукте в течение рассматриваемого периода (год, квартал), ед.;

$C_{o}$ - затраты на выполнение одного заказа, сум.;

$S$ - искомая величина заказа, ед.

Затраты на хранение партии продукции рассчитываются по формуле

$C_{x}=0,5 * C_{n}^{*} f^{*} S$,

где $C_{n}$ - цена единицы продукции, хранимой на складе, сум.;

$f$ - коэффициент, отражающий затраты на хранение запаса в виде доли от цены $C_{n}$.

Поскольку в формуле (3) учитывается средняя величина запаса, хранящегося на складе за период $T$, то вводится коэффициент 0,5.

Подставив выражения (2) и (3) в формулу (1), получим

$$
C_{\Sigma}=\frac{A * C_{0}}{S}+0,5 * C_{n} * f * S \rightarrow \min
$$

Следует подчеркнуть, что при выводе формулы (4) сделаны следующие допущения:

- затраты на выполнение заказа $C_{o}$, цена поставляемой продукции $C_{n}$ и затраты на хранение единицы продукции в течение рассматриваемого периода постоянны; 
Таблица 1. График зависимости затрат на заказ, хранение запаса и общих затрат от размера заказа

\begin{tabular}{|c|c|c|c|c|}
\hline № п/п & $S$ & $C_{3}$ & $C_{x}$ & $C_{\Sigma}$ \\
\hline 1 & 30 & 1333333 & 72000 & 1405333,3 \\
\hline 2 & 50 & 800000 & 120000 & 920000 \\
\hline 3 & 70 & 571428,6 & 168000 & 739428,57 \\
\hline 4 & 90 & 444444,4 & 216000 & 660444,44 \\
\hline 5 & 110 & 363636,4 & 264000 & 627636,36 \\
\hline 6 & 130 & 307692,3 & 312000 & 619692,31 \\
\hline 7 & 150 & 266666,7 & 360000 & 626666,67 \\
\hline 8 & 170 & 235294,1 & 408000 & 643294,12 \\
\hline 9 & 190 & 210526,3 & 456000 & 666526,32 \\
\hline 10 & 210 & 190476,2 & 504000 & 694476,19 \\
\hline 11 & 230 & 173913 & 552000 & 725913,04 \\
\hline 12 & 250 & 160000 & 600000 & 760000 \\
\hline 13 & 270 & 148148,1 & 648000 & 796148,15 \\
\hline 14 & 290 & 137931 & 696000 & 833931,03 \\
\hline 15 & 310 & 129032,3 & 744000 & 873032,26 \\
\hline 16 & 330 & 121212,1 & 792000 & 913212,12 \\
\hline 17 & 350 & 114285,7 & 840000 & 954285,71 \\
\hline 18 & 370 & 108108,1 & 888000 & 996108,11 \\
\hline 19 & 390 & 102564,1 & 936000 & 1038564,1 \\
\hline 20 & $\ldots$ & $\ldots$ & $\ldots$ & $\ldots$ \\
\hline
\end{tabular}

период между заказами (поставками) постоянный, т.е. $T=$ const;

- заказ $\mathrm{S}_{o}$ выполняется полностью, мгновенно;

интенсивность спроса $X=S_{o} / T$ постоянна;

- емкость склада не ограничена;

- рассматриваются только текущие (регулярные) запасы, другие виды запасов (страховые, подготовительные, сезонные, транзитные и т.д.) не учитываются.

Для определения минимального значения функции $C_{\Sigma}$ воспользуемся известной математической процедурой, а именно возьмем первую производную и приравняем ее нулю:

$$
\frac{d C_{\Sigma}}{d s}=-\frac{C_{o} * A}{s^{2}}+0,5 * C_{n} * f=0,
$$

Выполнив соответствующие преобразования в уравнении (5) находим оптимальную величину размера заказа EOQ (формула Уилсона):

$$
S_{o}=\sqrt{\frac{2 * C_{o} * A}{C_{n} * f}}
$$

Теперь необходимо определить остальные параметры, характеризующие модель EOQ.
Количество поставок за рассматриваемый период определим по формуле:

$$
N=\frac{A}{S_{o}}=\sqrt{\frac{A * C_{n} * f}{2 * C_{o}}},
$$

Продолжительность одного цикла определим по формуле:

$$
T=\frac{Z}{N}=\not * \sqrt{\frac{2 * C_{0}}{A * C_{n} * f^{\prime}}}
$$

где Д- рассматриваемый период, дн.

При расчетах необходимо учитывать, если вычисления будут выполняться в общем для всего годового периода, тогда Д = 365 дн.; при выполнении вычислений для рабочих дней в году то Д = $Д_{p}=260$ дн., если расчеты выполняются для недельного периода, тогда Д=Д $=52$ нед.

Определим величину минимальных общих затрат, путем подстановки $S_{o}$, рассчитанной по формуле (6), в уравнение (4) и выполнив соответствующие преобразования получим:

$$
C_{\Sigma \min }=\sqrt{2 * C_{o} * A * C_{n} * f} .
$$




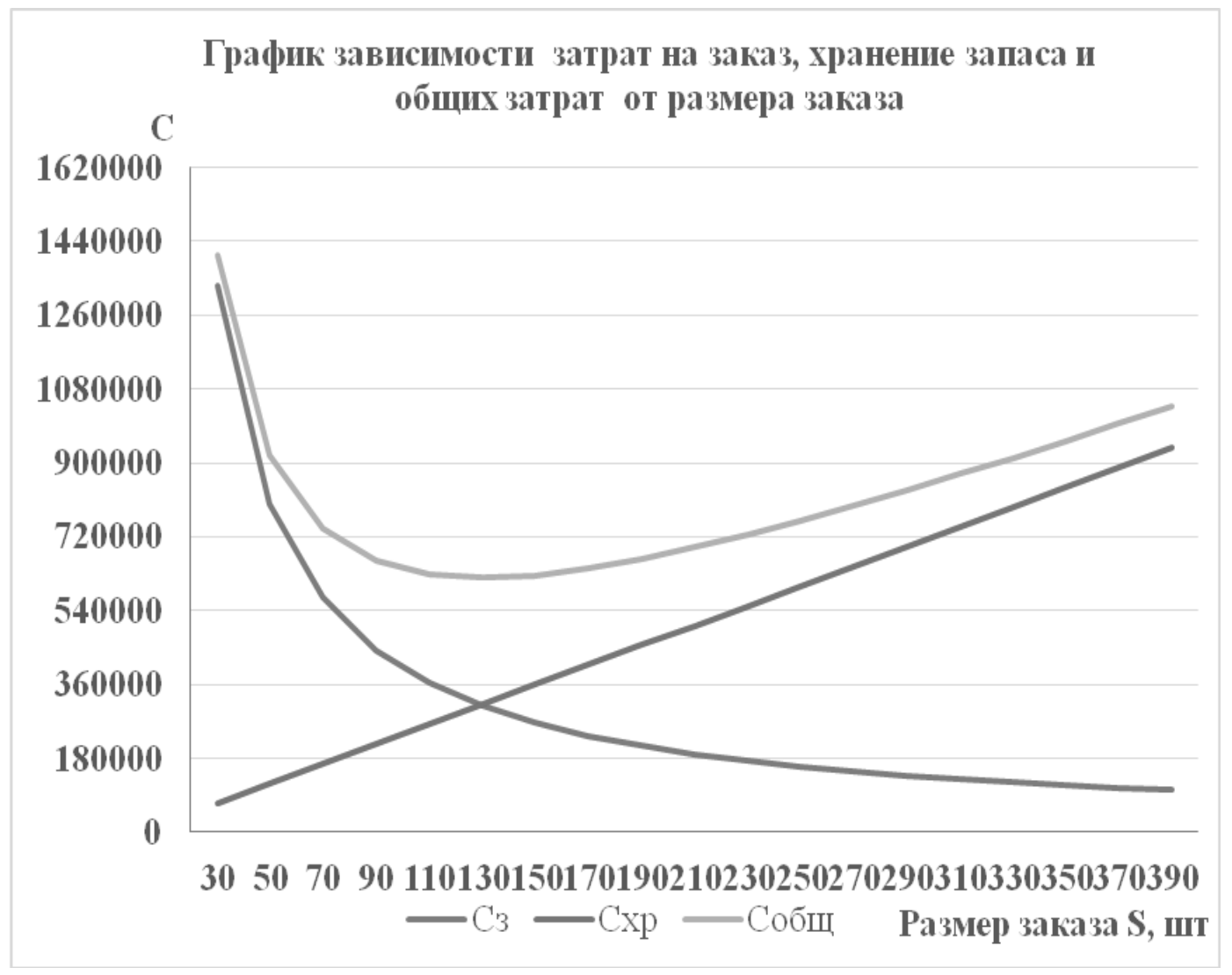

Рис. 1. Зависимость затрат на заказ Сз, хранение запаса Схр и общих затрат Собщ от размера заказа.

Формула (9) позволяет выполнить расчет минимальных затрат с учетом затрат на выполнение заказов $C_{3}$ и затрат на хранение запаса на складе $C_{x}$.

Выполним апробацию полученных формул с помощью исходных данных следующего примера.

\section{1-пример}

Рассчитать параметры модели оптимального размера запаса EOQ для следующих исходных данных: пусть запас ТМЦ на складе характеризуется тем, что он будет поступать циклически начиная 30 усл.ед. груза с шагом 20; цена единицы продукта $C_{n}=24000$ сум.; коэффициент, отражающий затраты на хранение ТМЦ примем равным $f=0,2$; затраты на выполнение одного заказа $C_{o}=80000$ сум.; расчетный период $Д_{p}=260$ дн.; потребность в заказываемом продукте (в год) $A=500$ ед.

Фрагмент результатов расчетов затрат на запас, хранение и общих затрат ТМЦ выполненных с помощью формул (2) - (4) приведены в табл. 1.

Анализ таблицы показывает, что минимум общих затрат наблюдается при значении S равном 130 усл.ед.
На основе данных табл. на рис. 1 приведены графики затрат на запас ТМЦ, хранение и общих затрат при изменяющемся их количестве $S$ (колеблется от 30 до 500 усл.ед.).

Теперь определим оптимальный размер заказа, воспользовавшись формулой (6) и сравним полученные результаты:

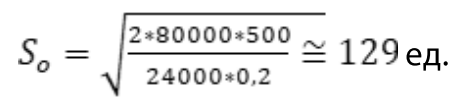

Таким образом можно заключить, что оптимальный размер запаса ТМЦ при необходимом максимальном количестве равном 500 усл.ед. составляет 129 ед.

Определим минимальные суммарные затраты на выполнение заказов и хранение ТМЦ в течение года, подставив численные значения в формулу (9):

$$
\begin{aligned}
& C_{\text {Imin }}=\sqrt{2 * C_{o} * A * C_{n} * f}= \\
& =\sqrt{2 * 80000 * 500 * 24000 * 0,2}=619677,34 \text { сум. }
\end{aligned}
$$

Полученный результат показывает, что 619692,31$619677,34=14,97$ сум отличается, т.е. всего на 0,0024\%. 
Количество заказов за рассматриваемый период согласно формулы (7) равно:

$$
N=\sqrt{\frac{500 * 24000 * 0,2}{2 * 80000}}=\sqrt{15} \cong 4 \text { заказа }
$$

Продолжительность одного цикла, т.е. периодичность выполнения заказов согласно формулы (8) равно:

$$
T=260 * \sqrt{\frac{2 * 80000}{500 * 24000 * 0,2}} \cong 17 \text { дней }
$$

Несмотря на соблюдение всех ограничений, допущения, принятые при выводе формулы Уилсона, требуют уточнения способов определения затрат, в первую очередь затрат на хранение. Расчеты затрат на хранение ТМЦ на складах вагонного депо показывают, что при этом, как правило, учитывается не средний размер партии, а площадь (или объем) склада, которая требуется для всей поступившей партии:

$$
C_{x}=\alpha * k * \theta * S
$$

где $\alpha$ - затраты на хранение продукции в единицу времени с учетом занимаемой площади (объема) склада, сум $/ \mathrm{M}^{2}{ }^{*}$ ед. времени (сум $/ \mathrm{m}^{3}{ }^{*}$ ед. времени); $k$ - коэффициент, учитывающий пространственные габариты единицы продукции, м²/шт (м³/шт); $\theta-$ коэффициент, учитывающий неодновременность поступления различных видов продукции на склад, $0<\theta<1$. Часто в расчетах $\theta$ принимают равным $1(\theta=1)$. Теперь подставив выражение (10) в формулу (4), получим (при $\theta=1)$

$$
C_{\Sigma}=\frac{A * C_{0}}{S}+\alpha * \mathrm{k} * \mathrm{~S} \rightarrow \min ,
$$

С целью определения минимального значения функции $C_{\Sigma}$ как было выше отмечено возьмем первую производную и приравняем ее нулю, а также выполнив последующие соответствующие преобразования, определим оптимальный размер заказа:

$$
S_{o}=\sqrt{\frac{c_{o} * A}{\alpha * k}} .
$$

Подставив $\mathrm{S}_{o}$ из формулы (12) в формулу (11) выполнив соответствующие преобразования получим выражение позволяющее вычислить величину минимальных затрат, которая учитывает затраты на хранение продукции в единицу времени с учетом занимаемой площади:

$$
C_{\min }=2 * \sqrt{C_{o} * A * \alpha * k}
$$

Выполним апробацию полученных формул с помощью исходных данных предыдущего, т.е. 1-примера.

\section{2-пример}

Предположим, что каждая единица продукции упакована в ящик следующих размеров: $a \times 8 \times c(a=$ 0,3 м - ширина; $8=0,4$ м - длина; $c=0,3$ м - высота); при хранении допускается штабелирование ящиков в $А$ ярусов $(A=6)$.

Рассчитаем затраты на хранение единицы продукции при условии, что выбран склад класса В (теплое помещение, первый этаж), т.е. ставка 9,0 усл. ед. $/$ м $^{2}{ }^{*}$ мес.

Найдем величины $\alpha$ и $k$ при условии 1 усл. ед. $=260$ сум.:

$$
\begin{aligned}
& \alpha=9,0 * 12 * 260=28100 \text { сум } / \mathrm{M}^{2 *} \text { год; } \\
& k=\frac{\alpha * b}{h}=\frac{0,3 * 0,4}{6}=0,02 \mathrm{M}^{2} / \text { ед. }
\end{aligned}
$$

Следовательно,

$$
\alpha^{*} k=28100 * 0,02=560,2 \text { сум/ед. * год. }
$$

Оптимальный размер заказа равен

$$
S_{o}=\sqrt{\frac{80000 * 2000}{560,2}} \cong 535 \text { ед., }
$$

минимальные суммарные затраты

$$
C_{\text {Emin }}=2 * \sqrt{80000 * 2000 * 560,2}=598772 \text { сум }
$$

Соответственно, количество заказов $N \cong 6$ заказа, а периодичность заказов $T \cong 5$ дней.

Расчет общих затрат, выполненный по методике 2-го примера, является более точным и экономичным.

Надо отметить, что анализ складов вагонного депо ВчД-2 показал, что получаемые ТМЦ хранятся без штабелирования, размещаются по товарным группам на поддонах. Следовательно, для определения оптимального размера заказа ТМЦ в ВЧД-2 нами предлагается использовать формулы (2) - (9).

\section{Зак^ючение}

1. Расчет размера запаса на складе с учетом площади (или объема) склада, позволяет получить более точные результаты общих расходов на покупку и хранение ТМЦ.

2. Оптимальную величину размера заказа $\mathrm{S}_{o} \mathrm{TMЦ,}$ комплектующих и запасных частей подвижных единиц вагонного депо предлагается выполнять, используя формулу (6).

3. Определив оптимальный размер заказа $\mathrm{S}_{o}$ можно определить количество заказов и продолжительность одного цикла, которые составили 
соответственно 4 заказа при периодичности равной 17 дням (формулы 7 и 8).

Определены формулы расчета оптимального размера запаса $\mathrm{C}_{3}$ оптимального количества заказов за период (частота завоза) $N_{\text {опт }}$ оптимальный период между поставками $T_{\text {onm }}$.
Приведенные формулы позволяют: оптимизировать запасы ТМЦ на складе вагонного депо, повысить качество и уровень материально-технического обеспечения производственного цикла ремонтно-экипировочных работ, выполняемых с подвижными единицами, оптимизировать размеры партии поставок продукции.

\section{ЛИТЕРАТУРА}

1. Нурмухамедов Т.Р., Гулямов Ж.Н. Разработка базы данных учета складского инветаря вагонного депо. // Сборник научных статей по итогам одиннадцатой международной научной конференции: “Передовые инновационные разработки. Перспективы и опыт использования, проблемы внедрения в производство", часть 2.-Казань, 2019.-С.212-215.

2. Управление запасами в цепях поставок: Учеб. пособие; под общ. и научн. ред. В.С. Лукинского. — СПб.: СПбГИЭу, 2010. — 372 с.

3. Логистика: учебник / Под ред. Б.А. Аникина. - М.: ИНФРА-М, 2008. - 368 с.

4. Гаджинский, А.М. Управление запасами в логистике / А.М. Гаджинский // Справочник экономиста. — 2008, № 2. - C. 66-77.

○ Нурмухамедов Толаниддин Рамзиддинович ( ntolaniddin@mail.ru ), Гулямов Жавлон Нуруллаевич ( javlonbek1207@gmail.com ), Абдуллаев Элдор Саъдулла угли ( eldorabdullayev@list.ru ), Азимов Абдулхай Абдулатиф угли ( azimovabdulhay1707@gmail.com ). Журнал «Современная наука: актуальные проблемы теории и практики»

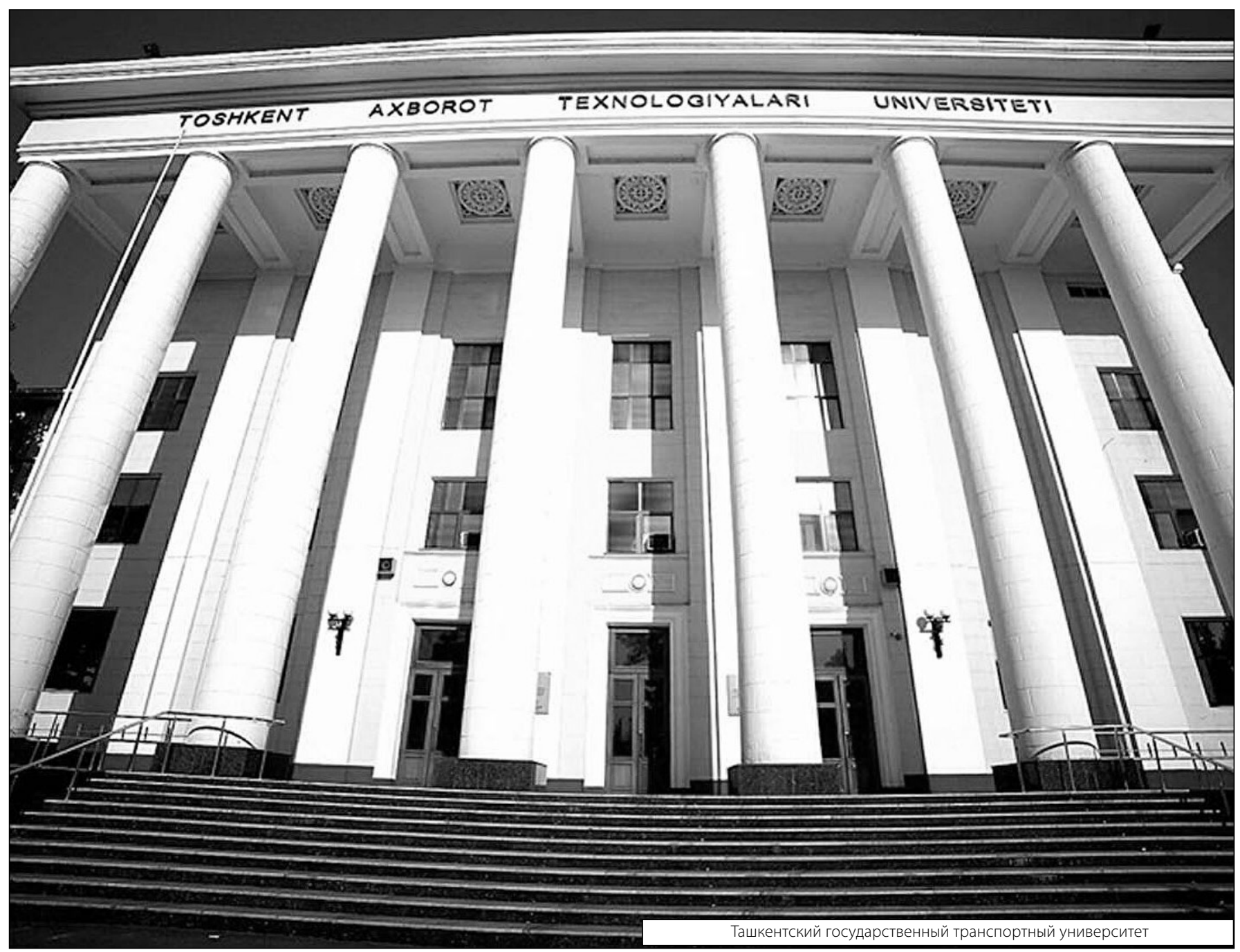

